INPLASY

PROTOCOL

To cite: Buczyłowska et al.

Exposure to greenspace and bluespace and cognitive functioning in children - a systematic review protocol. Inplasy protocol 202220018. doi:

10.37766/inplasy2022.2.0018

Received: 08 February 2022

Published: 08 February 2022

Corresponding author:

Dorota Buczyłowska

dorota.buczylowska@uj.edu.pl

Author Affiliation: Institute of Psychology, Jagiellonian University.

Support: Foundation for Polish Science.

Review Stage at time of this submission: Formal screening of search results against eligibility criteria.

Conflicts of interest:

None declared.

\section{Exposure to greenspace and bluespace and cognitive functioning in children - a systematic review protocol}

Buczyłowska, D1; Zhao, T2; Singh, N33; Jurczak, A4; Siry, A5; Markevych, ${ }^{6}$.

Review question / Objective: Following review questions should be addressed: Is there any association between exposure to greenspace or bluespace and cognitive functioning in children as assessed by observational and interventional research? Does exposure to greenspace or bluespace is more strongly associated with a particular domain of cognitive functioning in children compared to other domains of cognitive functioning? Does the association between exposure to greenspace or bluespace and cognitive functioning in children differ according to age? Does the association between greenspace and bluespace differ depending on type and methods of the exposure assessment? Information sources: PubMed and PsycINFO via EBSCO will be searched. We will also conduct a 'snowball' search to detect additional studies by searching the reference lists of publications eligible for full-text review. Further, we will manually search reference lists of previously published reviews.

INPLASY registration number: This protocol was registered with the International Platform of Registered Systematic Review and Meta-Analysis Protocols (INPLASY) on 08 February 2022 and was last updated on 08 February 2022 (registration number INPLASY202220018).

\section{INTRODUCTION}

Review question / Objective: Following review questions should be addressed: Is there any association between exposure to greenspace or bluespace and cognitive functioning in children as assessed by observational and interventional research?
Does exposure to greenspace or bluespace is more strongly associated with a particular domain of cognitive functioning in children compared to other domains of cognitive functioning? Does the association between exposure to greenspace or bluespace and cognitive functioning in children differ according to 
age? Does the association between greenspace and bluespace differ depending on type and methods of the exposure assessment?

Rationale: The field of green- and bluespace research is rapidly developing, and there are more and more studies in relation to cognitive outcomes. Hence, there is a strong need to systematically review the existing studies and to detect research gaps. Seven systematic reviews have been already published in relation to this topic (de Keijzer et al. 2016; Gascon et al. 2015; Islam et al. 2020; Luque-García et al. 2021; Ohly et al. 2016; Stevenson et al. 2018; Vanaken \& Danckaerts 2018) and four systematic reviews are currently registered (Ferguson et al.; Herchet et al.; VellaBrodrick \& Gilowska; Xiao-Ling et al.) but none of them is specific to cognitive outcomes in children of the entire age range. Moreover, only Gascon et al. (2015) looked into the effects of bluespace in addition to greenspace. Also, except for the Gascon et al. (2015), published or planned reviews are focused either only on observational studies (Islam et al. 2020; de Keijzer et al. 2016; Ferguson et al.; LuqueGarcía et al. 2021; Vanaken \& Danckaerts 2018) or only on experimental studies (Ohly et al. 2016; Stevenson et al. 2018; VellaBrodrick \& Gilowska; Herchet et al.). The registered review by Xiao-Ling et al. will only summarize studies which investigated diagnosed neurodevelopmental disorders. Finally, nearly none of these reviews, except for Islam et al. 2020; Stevenson et al. (2018) and Herchet et al., conducted search in Psychlnfo database, which lists environmental psychological research that is not necessarily indexed by PubMed or Web of Science. Our systematic review will be focused on cognitive outcomes in relation to green- and bluespace in children of all ages. We will conduct search in two major databases - PubMed and PsychInfo, to capture observational and experimental studies from the fields of environmental epidemiology and environmental psychology. We believe that our interdisciplinary team of environmental epidemiologists and psychologists who are specialised in green- and bluespace, cognition, and systematic reviews, will produce a high-quality systematic review on the topic.

Condition being studied: Not applicable.

\section{METHODS}

Search strategy: Searches were conducted on two data bases - PubMed and Psyclnfo, both on 17.12.2021. Free-text terms related to outcome (e.g. cognition, cognitive, intelligence), exposure (e.g. greenspace, green space, greenness), and population (e.g. child, boy, girl) as well as Medical Subject Headings (MeSH) terms for outcome (e.g. cognition, intelligence) and population (e.g. child, adolescent) were used. We will also conduct a 'snowball' search to detect additional studies by searching the reference lists of publications eligible for full-text review.

Participant or population: The target population is children up to 18 years of age, boys and girls, regardless of ethnicity. Exclusion: Neurodevelopmental disorders such as ADHD and autism spectrum disorder; any clinical condition involving brain damage; age above 18 years of age; animal studies.Studies containing a mixed population will be included when some of the participants meet the eligibility criteria.

Intervention: Not applicable.

Comparator: Children exposed to lower greenspace or bluespace levels.

Study designs to be included: Cohort studies or longitudinal studies or casecontrol studies or cross-sectional or experimental studies. Excluded are studies focused on behaviour only (ie, without cognition). There are no restrictions on the basis of the date of publication.

Eligibility criteria: Eligibility criteria according to the PECOS (Population, Exposure, Comparator, Outcome, Study) statement:(P) normal human population of children up to 18 years of age; (E) assessed greenspace or bluespace exposure using an objective measure (including NDVI, 
proportion of greenspace or blue space, distance to greenspace or blue space), or subjective measures (including selfreported frequency of visits to greenspaces or blue spaces, quality of green space or blue space);(C) exposed to higher greenspace or bluespace compared to those exposed to lower levels; (0) assessed cognitive functioning using an objective measure such as standardized psychometric tasks(S) original human studies including cohort studies or longitudinal studies or case-control studies or cross-sectional, quasi-experimental or experimental studies, published in a peerreviewed scientific journal. Excluded are studies focused on behavior only (ie, without cognition). There are no restrictions on the basis of the date of publication.

Information sources: PubMed and PsycINFO via EBSCO will be searched. We will also conduct a 'snowball' search to detect additional studies by searching the reference lists of publications eligible for full-text review. Further, we will manually search reference lists of previously published reviews.

Main outcome(s): Main outcome will be cognitive functioning including such domains as attention, memory, executive functioning, and intelligence etc. The outcomes will be extracted if they were measured using standardized psychometric tasks designed to assess cognitive functioning. If multiple outcomes are reported, only those derived from cognitive measures will be extracted. If multiple outcomes assessing different cognitive domains are reported, all of them will be extracted and accordingly assigned to specific cognitive domains.

\section{Additional outcome(s): Not applicable.}

Data management: Two independent reviewers will scan titles and abstracts using scanning software Rayyan (https:// www.rayyan.ai/) and they will further review the full-text publications to check for their eligibility for inclusion. If there is any difference in the eligibility of a publication between the two reviewers, it will be resolved by a discussion with/decision of a third reviewer. Two independent reviewers will conduct data extraction. The reviewers will control each other' extractions and confirm their accuracy. An excel spreadsheet will be used for data extraction. The items to be extracted relating to the study characteristics are as follows: the report (author(s), year of publication, country); sample characteristics (age, type of school/type of population, sample size); study design, exposure measures, cognitive outcome measures, and results. Any discrepancies in data extraction between the two reviewers will be resolved by discussion with a third reviewer.

Quality assessment / Risk of bias analysis: We will evaluate the risk of bias (ROB) according to the Office of Health Assessment and Translation (OHAT) tool by the National Institutes of Environmental Health Sciences National Toxicology Program (OHAT, 2015) and Navigation Guide by the University of California (Lam et al., 2016; Woodruff and Sutton, 2014) for each included study. In light of the OHAT tool, we will assess Key criteria (Exposure assessment, Outcome assessment, Confounding bias) and Other Criteria (Selection bias, Attrition/exclusion bias, Selective reporting bias, Conflict of interest, Other source of bias). Each of the above domains should be evaluated as "low", "probably low", "probably high", or "high" risk based on specific criteria. Consequently, the studies for which the key criteria and most of the other criteria are characterized as "high" or "probably high" risk will be removed from our included study pool.

Strategy of data synthesis: Not applicable.

Subgroup analysis: Not applicable.

Sensitivity analysis: Not applicable.

Language: Language restriction will be imposed in the search strategy, publications only in English, German, and Polish will be selected. 
Country(ies) involved: Poland and Germany.

Other relevant information: The second affiliation: Institute and Clinic for Occupational, Social and Environmental Medicine, LMU University Hospital Munich, Munich, Germany.

Keywords: cognition; cognitive functioning; greenspace; green space; greenness; greenery; bluespace; blue space; children.

Dissemination plans: The systematic review results will be disseminated by publication in a peer-reviewed journal and presenting at scientific conferences.

\section{Contributions of each author:}

Author 1 - Dorota Buczyłowska conceiving the review; designing the review; coordinating the review; data collection; data management; screening of data; extraction of data; interpretation of data; writing the protocol; writing the review.

Email: dorota.buczylowska@uj.edu.pl

Author 2 - Tianyu Zhao - conceiving the review; designing the review; analysis of data, writing the protocol; writing the review.

Email: tianyu.zhao@med.uni-muenchen.de Author 3 - Nitika Singh - conceiving the review; extraction of data; interpretation data; analysis of data; writing the review.

Email: nitika.singh@doctoral.uj.edu.pl

Author 4 - Agnieszka Siry - collection and screening of data.

Email: a.siry@student.uj.edu.pl

Author 5 - Anna Jurczak - collection and screening of data.

Email: anna.jurczak@student.uj.edu.pl

Author 6 - lana Markevych - conceiving the review; designing the review; screening of data, extraction of data; analysis of data; interpretation data; writing the protocol; writing the review.

Email: iana.markevych@uj.edu.pl

\section{References:}

de Keijzer, C., Gascon, M., Nieuwenhuijsen, M. J., \& Dadvand, P. (2016). Long-term green space exposure and cognition across the life course: a systematic review. Current Environmental Health Reports, 3,
468-477. https://doi.org/10.1007/ s40572-016-0116-x.

Ferguson, M., Wheeler, B., Teyhan, A., McEachan, R., Lovell, R. \& Lawal, H., M. The influence of greenspace exposure on cognitive, emotional, social and behavioural development: a systematic review of longitudinal studies in childhood and adolescence. PROSPERO 2019 CRD42019143134. https: / / w w w.crd.york.ac.uk/prospero/ display_record.php?ID=CRD42019143134.

Gascon, M., Mas, M. T., Martínez, D., Dadvand, P., Forns, J., Plasència, A., \& Nieuwenhuijsen, M. J. (2015). Mental health benefits of long-term exposure to residential green and blue spaces: $A$ systematic review. International Journal of Environmental Research and Public Health, 12, 4354-4379.

Herchet, M., Hofmann, M., Venený, M. The Growing Evidence - A Meta-analysis of (quasi-) experimental data on the mental health benefits of urban greenspace. PROSPERO 2021 CRD42021245057. https:// w w w . crd.york. a c . uk/prospero/ display_record.php?ID=CRD42021245057.

Islam, M. Z., Johnston, J., \& Sly, P. D. (2020). Green space and early childhood development: A systematic review. Reviews on Environmental Health, 35, 189-200. https://doi.org/10.1515/REVEH-2019-0046.

Lam, J., Sutton, P., Kalkbrenner, A., Windham, G., Halladay, A., Koustas, E., Lawler, C., Davidson, L., Daniels, N., Newschaffer, C., \& Woodruff, T. (2016). A systematic review and meta-analysis of multiple airborne pollutants and autism spectrum disorder. PLoS One, 11. https:// doi.org/10.1371/journal.pone.0161851.

Luque-García, L., Corrales, A., Lertxundi, A., Díaz, S., \& Ibarluzea, J. (2021). Does exposure to greenness improve children's neuropsychological development and mental health? A navigation guide systematic review of observational evidence for associations. Environmental 
Research, 206, 112599. https://doi.org/ 10.1016/j.envres.2021.112599.

OHAT, 2015. Translation (OHAT) Division of the National Toxicology Program National Institute of Environmental Health Sciences.

Stevenson, M. P., Schilhab, T., \& Bentsen, P. (2018). Attention restoration theory II: a systematic review to clarify attention processes affected by exposure to natural environments. Journal of Toxicology and Environmental Health - Part B: Critical Reviews, 21, 227-268. https://doi.org/ 10.1080/10937404.2018.1505571.

Vanaken, G. J., \& Danckaerts, M. (2018). Impact of green space exposure on children's and adolescents' mental health: A systematic review. International Journal of Environmental Research and Public Health, 15. https://doi.org/10.3390/ ijerph15122668.

Vella-Brodrick, D. \& Gilowska, K. Does access to nature (greenspace) impact cognitive functioning in children and adolescents? A systematic review. PROSPERO 2021 CRD42021214826.

https://www.crd.york.ac.uk/prospero/ display_record.php?ID=CRD42021214826.

Woodruff, T. J., \& Sutton, P. (2014). The navigation guide systematic review methodology: A rigorous and transparent method for translating environmental health science into better health outcomes. Environmental Health Perspectives, 122, 1007-1014. https://doi.org/10.1289/ ehp.1307175.

Xiao-Ling, Z., Li-Zi, L., Jin, J. \& Guang-Hui, D. A systematic review of the associations between outdoor greenspace and neurodevelopmental disorders. PROSPERO 2021 CRD42021260901. https:// w w w. crd.york.ac.uk/prospero/ display_record.php?ID=CRD42021260901. 